\title{
Scepticaemia: The impact on the health system and patients \\ of delaying new treatments with uncertain evidence; a case
}

\section{study of the sepsis bundle [version 1; peer review: 2 approved]}

\author{
Robin Blythe (D1, David Cook², Nicholas Graves (D1) \\ ${ }^{1}$ Australian Centre for Health Services Innovation, School of Public Health and Social Work, Institute for Health and Biomedical \\ Innovation, Queensland University of Technology, Brisbane, Queensland, Australia \\ ${ }^{2}$ Intensive Care Unit, Princess Alexandra Hospital, Brisbane, Queensland, Australia
}

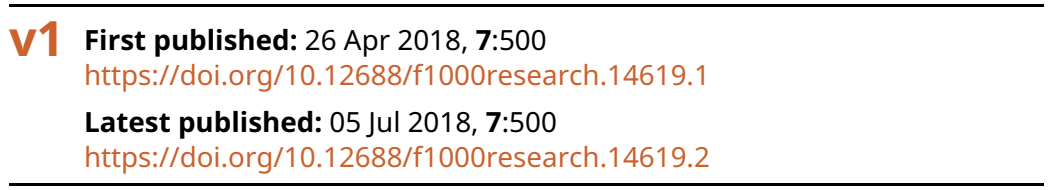

\section{Abstract}

Background: A sepsis care bundle of intravenous vitamin C, thiamine, and hydrocortisone was reported to improve treatment outcomes.

The data to support it are uncertain and decision makers are likely to be cautious about adopting it. The objective of this study was to model the opportunity costs in dollars and lives of waiting for better information before adopting the bundle.

Methods: A decision tree was built using information from the literature. We modelled the impact of bundle adoption under three scenarios using a simulation in which the bundle was effective as reported in the primary trial, less effective based on other information, and ineffective.

The measurements were health services costs, quality-adjusted life years, and transition probabilities.

Results: If the bundle proves to be effective under either scenario, it will save billions of dollars and millions of life-years in the United States. This is the opportunity cost of delaying an adoption decision and waiting for better quality evidence. We suggest that hospital decision-makers consider implementing the bundle on a trial basis while monitoring costs and outcomes data even while the evidence base is uncertain.

Conclusions: If the decision maker is unwilling to use the best available evidence now, but rather wishes to wait for definitive evidence they are risking incurring large costs for health care systems and for the patients they serve. An explicit analysis of uncertain clinical outcomes is a useful adjunct to guide decision making where there is clinical ambiguity. This approach offers a valid alternative to the default of clinical inactivity when faced with uncertainty.

\section{Keywords}

Sepsis, Costs and Cost Analysis, Evidence-based practice

\section{Open Peer Review}

Approval Status

1 2

version 2

(revision)

05 Jul 2018

version 1

26 Apr 2018

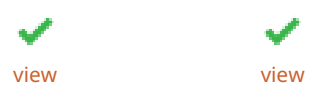

1. Paul E. Marik, Eastern Virginia Medical School, Norfolk, USA

2. Adam Visser, Toowoomba Hospital,

Toowoomba, Australia

Any reports and responses or comments on the article can be found at the end of the article. 
Corresponding author: Robin Blythe (robin.blythe@qut.edu.au)

Author roles: Blythe R: Conceptualization, Formal Analysis, Investigation, Methodology, Project Administration, Validation, Visualization, Writing - Original Draft Preparation, Writing - Review \& Editing; Cook D: Conceptualization, Methodology, Writing - Review \& Editing; Graves N: Conceptualization, Formal Analysis, Methodology, Supervision, Writing - Original Draft Preparation, Writing Review \& Editing

Competing interests: No competing interests were disclosed.

Grant information: The author(s) declared that no grants were involved in supporting this work.

Copyright: ( 2018 Blythe R et al. This is an open access article distributed under the terms of the Creative Commons Attribution License, which permits unrestricted use, distribution, and reproduction in any medium, provided the original work is properly cited. Data associated with the article are available under the terms of the Creative Commons Zero "No rights reserved" data waiver (CC0 1.0 Public domain dedication).

How to cite this article: Blythe R, Cook D and Graves N. Scepticaemia: The impact on the health system and patients of delaying new treatments with uncertain evidence; a case study of the sepsis bundle [version 1; peer review: 2 approved] F1000Research 2018, 7:500 https://doi.org/10.12688/f1000research.14619.1

First published: 26 Apr 2018, 7:500 https://doi.org/10.12688/f1000research.14619.1 


\section{Introduction}

Sepsis arises frequently among patients admitted to hospitals in the US and elsewhere. It is often fatal, accounting for $30 \%$ to $50 \%$ of inpatient deaths ${ }^{1}$. Those who survive incur large costs from increased risk of organ damage ${ }^{2}$. A recent paper by Marik et al. (2017) described the effectiveness of a treatment bundle made up of intravenous vitamin $\mathrm{C}$, thiamine and hydrocortisone $^{3}$. Because of small sample size, non-randomised design, large observed treatment effects and the simple and low cost characteristics of the bundled intervention, there was scepticism among clinicians, administrators and payers regarding adoption ${ }^{4-6}$. Rapid adoption without good evidence has risks and costs, yet delaying an effective treatment imposes larger costs to patients and the health system. Waiting for conclusive evidence might be a poor and costly strategy and should be balanced against the likely costs of rapid adoption with uncertain and low quality evidence.

The aim of this paper is to estimate the economic consequences of a decision to adopt the Marik sepsis bundle early, under conditions of large uncertainty. This is compared to the alternative, which is to wait one year; the time period between publication of the first paper in June $2017^{3}$ and the completion of the National Institutes of Health funded trial due in mid-2018. The trial will ideally reduce the uncertainty in an adoption decision. This paper predicts the cost savings and health benefits of adopting the Marik bundle immediately with uncertain evidence for the entire United States health system, compared to waiting for better quality evidence. Our estimates examine likely changes to costs and health outcomes if the treatment is found to be effective, less effective and ineffective.

\section{Methods}

An incremental cost-effectiveness analysis was conducted on the change to costs and Quality-Adjusted Life Years (QALYs) of standard sepsis care compared to the early adoption of the Marik bundle ${ }^{3}$. Values for expected costs and health utilities were taken from the literature, with probabilities of treatment outcomes taken from Marik's paper ${ }^{3}$. The time horizon was 5 years in order to sufficiently measure the long-term outcomes of acute kidney injury (AKI), an important and common result of severe septic shock ${ }^{2}$. A decision tree was programmed in TreeAge Pro 2017 R2.1 (Williamstown, MA2017) and prior statistical distributions of costs, outcomes and probabilities used to include uncertainties in the data, see Figure 1. Patients receiving either modality progress through a chance node to death or survival. Surviving patients progress through a second chance node, where they would either recover or recover with organ damage. AKI is a common outcome in cases of sepsis, and typically require renal replacement therapy, which can take the form of either dialysis or organ transplantation ${ }^{2}$.

\section{Costs}

The costs of care include the hospital cost of a sepsis episode, the additional cost of the Marik treatment bundle and the annual ongoing cost of renal replacement therapy (RRT). Hospital costs were taken from a systematic review by Arefian et al. (2017) for a US perspective with a mean of $\$ 32,421$ and standard deviation of $\$ 15,051$ per stay ${ }^{7}$. For the Marik bundle, we used an estimate of $\$ 528$ and a standard deviation of $5 \%$ at $\$ 26.50$ per patient per course. The annual cost of RRT for end stage renal disease varies depending on treatment modality, such as dialysis or transplant, and a weighted average of $\$ 76,936$, with a standard deviation of $\$ 15,387$ was taken from the 2017 USRDS Annual Data Report ${ }^{8}$.

\section{Health utilities}

The health utility score of patients reflects the value of their heath state and is a measure of health related quality of life. It is expressed on a range between zero, the worst health state, and one, the best possible health state ${ }^{9}$. The scores for patients who recover from septic shock depend on whether or not they suffered AKI. Patients who did not suffer AKI still underwent the stress of intensive care unit recovery, and their mean health utility scores

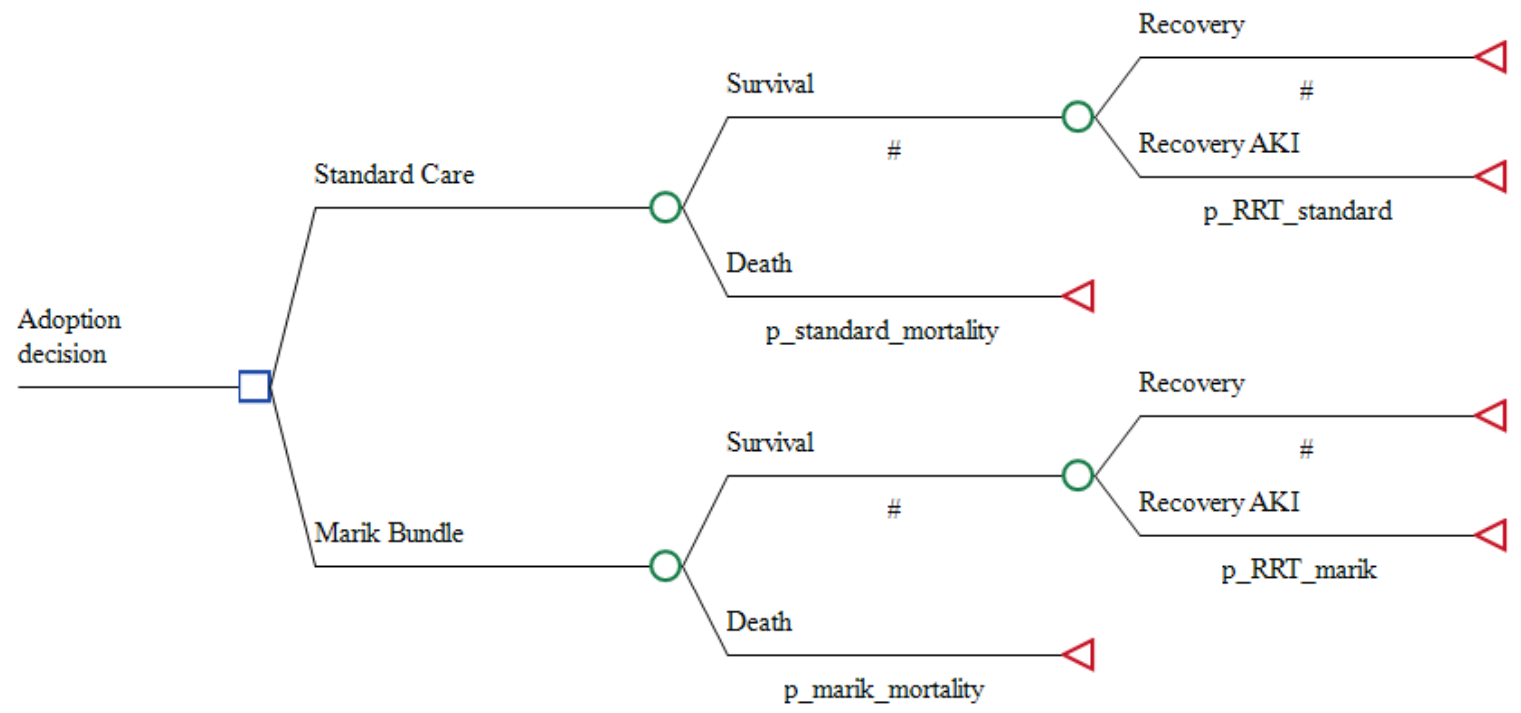

Figure 1. Sepsis decision tree model using TreeAge Pro 2017. 
and standard deviations were taken from Cuthbertson et al. (2010) at $1,2.5$, and 5 years as $0.666(0.280), 0.701$ (0.281), and $0.677(0.301)$, respectively ${ }^{10}$. Survivors of AKI were found to have a utility of 0.40 with a standard deviation of 0.37 at 60 days. We did not find evidence of improved health utilities over the 5 year period ${ }^{11}$. The health utility of death was valued at $0^{9}$.

\section{Transition probabilities}

The transition probabilities of patients moving through the decision tree were informed by data from Marik's study. This includes the probabilities of survival and AKI, see Table $1^{3}$. In Marik's study, the bundle's probability of death and AKI were 0.085 and 0.097 , with standard deviations of 0.041 and 0.043 respectively. These compared to the standard care mortality and AKI probabilities of 0.404 and 0.367 , with standard deviations of 0.071 and 0.070 respectively.

\section{Modelling additional scenarios}

Scenario 1 uses probabilities from the literature for mortality and AKI; costs and utilities were unchanged. The attributable mortality risk and rate of AKI in the general population vary in the literature and are different to those reported in Marik's control cohort. We used the mortality risk identified in Rhee et al. (2017) of $21.2 \%$ and the rate of sepsis-associated AKI in Angus et al. (2001) of $22.0 \%{ }^{12,13}$. These are lower than the rates seen in Marik's observation group. We estimated large standard deviations for mortality and AKI at $30 \%$ and $28 \%$ of the means respectively to include Marik's treatment group results as possible outcomes within $95 \%$ confidence intervals.

Scenario 2 represents a worst-case scenario, in which Marik's bundle is ineffective and there is no difference in probabilities between the bundle and standard care. We modelled scenario 2 using the same transition probabilities found in Rhee et al. and Angus et al. for both arms of the decision tree. As in scenario 1, costs and utilities remained unchanged.

\section{Probabilistic sensitivity analysis}

Probabilistic sensitivity analysis was conducted on the decision tree by taking 1000 random resamples of values from the prior statistical distributions of model parameters. This propagates all uncertainty in the data forward to the results and provides useful information for decision making.

\section{Results}

The total economic cost of patients under standard care, including 5 year estimations of RRT, was $\$ 116,427$ compared to the total economic cost of patients receiving the Marik bundle of $\$ 66,744$, an expected saving of $\$ 49,683$ per patient. The annual incidence of sepsis in the United States is $1.5 \mathrm{~m}$ cases suggesting a total expected cost saving of \$74.5bn USD. Over the same 5-year period, patients gain an additional 1.26 QALY per case, or $1.9 \mathrm{~m}$ QALYs per year for the population. Given the reduced costs and improved outcomes, the Marik bundle dominates standard care, as it both saves costs and increase health outcomes at the same time. The probabilistic sensitivity analysis shows this conclusion arises $98.0 \%$ of the time.

Scenario 1 reduces the mean expected economic cost of standard care by $\$ 17,166$ to $\$ 100,244$ per patient, compared to the Marik bundle at $\$ 67,581$. The expected cost saving between the bundle and standard care $\$ 32,663$ per patient, a total saving of $\$ 49.0 \mathrm{bn}$ USD, down from $\$ 74.7 \mathrm{bn}$. Altering the transition probabilities to values from the literature also reduces the QALYs gained, with patients gaining only 0.55 QALY per case, or 825,000 QALY per year over $1.5 \mathrm{~m}$ cases per year. In this scenario, the Marik bundle still dominates standard care by saving costs and improving health outcomes. The probabilistic sensitivity analysis shows this conclusion arises $90.8 \%$ of the time.

Scenario 2 is that there is no change to mortality outcomes and AKI between Marik's bundle and standard care. The mean cost per patient over 5 years is \$98,932USD for standard care and $\$ 99,459$ USD for the Marik bundle, an increase of \$527 per patient over a 5 year period. There is no difference in QALYs between treatment alternatives. We were unable to find any evidence of harm to the patient as a result of the bundle. Hydrocortisone and thiamine are already present in routine sepsis care, and the dosage of $6 \mathrm{~g}$ of vitamin $\mathrm{C}$ per day has been shown to be safe unless contraindicated $^{14-16}$. In this scenario, assuming the intervention is universally adopted, no patients will have been harmed, but healthcare payers and providers would have spent an additional $\$ 791 \mathrm{~m}$ USD per year. Outcomes from the scenario analyses are listed in Table 2 below.

Table 1. Transition probabilities from bundle decision to survival and recovery.

\begin{tabular}{|l|l|l|l|}
\hline & $\begin{array}{l}\text { Mean Standard } \\
\text { Care (SD) }\end{array}$ & $\begin{array}{l}\text { Mean Standard Care, } \\
\text { Scenario 1 (SD) }\end{array}$ & $\begin{array}{l}\text { Mean Marik } \\
\text { Bundle (SD) }\end{array}$ \\
\hline Mortality & $0.404(0.071)$ & $0.212(0.064)$ & $0.085(0.041)$ \\
\hline AKI & $0.367(0.070)$ & $0.220(0.062)$ & $0.097(0.043)$ \\
\hline
\end{tabular}


Table 2. Scenario analyses for adoption compared to baseline adoption outcomes.

\begin{tabular}{|l|l|l|l|l|}
\hline & $\begin{array}{l}\text { Cost } \\
\text { savings }\end{array}$ & $\begin{array}{l}\text { 95\% uncertainty } \\
\text { interval }\end{array}$ & $\begin{array}{l}\text { QALYs } \\
\text { gained }\end{array}$ & $\begin{array}{l}\text { 95\% uncertainty } \\
\text { interval }\end{array}$ \\
\hline Baseline & $\$ 74.5 \mathrm{bn}$ & {$[\$ 1.8 \mathrm{bn}-\$ 166.5 \mathrm{bn}]$} & $1.9 \mathrm{~m}$ & {$[0.3 \mathrm{~m}-3.6 \mathrm{~m}]$} \\
\hline Scenario 1: less effective & $\$ 48.5 \mathrm{bn}$ & {$[-\$ 22.7 \mathrm{bn}-\$ 132.4 \mathrm{bn}]$} & $0.8 \mathrm{~m}$ & {$[-0.2 \mathrm{~m}-2.1 \mathrm{~m}]$} \\
\hline Scenario 2: ineffective & $-\$ 0.791 \mathrm{bn}$ & {$[-\$ 0.9 \mathrm{bn}--\$ 0.7 \mathrm{bn}]$} & 0 & 0 \\
\hline
\end{tabular}

\section{Dataset 1. Sepsis Distributions Table}

http://dx.doi.org/10.5256/f1000research.14619.d201411

Data on distributions used in the analysis

\section{Discussion}

We found that adopting the Marik bundle has a high likelihood to save billions of dollars and generate millions of extra QALYs under the conditions outlined in Marik's paper and in an alternate scenario that uses other data. Under the ineffective treatment of scenario 2 costs are increased to health services by $\$ 0.791 \mathrm{bn}$. These results reveal substantial opportunity costs in dollars and lives if we fail to implement and the bundle is ultimately found to be effective, even if the treatment effect is lower than purported by Dr Marik. If the bundle does not work then some costs have been incurred by hospitals for no heath gain. Not adopting the bundle because the evidence for effectiveness is currently uncertain could well be a poor strategy.

The scenario analysis uses values at which the Marik bundle is less effective, specifically by aligning observation group figures with the literature. The mortality rate of sepsis under standard care was suggested by Marik to be $40.4 \%$. Sepsis mortality has been declining in the US, from $46.9 \%$ in the early 1990s to $21.2 \%$ in 2014 , declining by about $3 \%$ per annum and driven by improved organ support systems and protocoled early recognition and treatment ${ }^{13,17,18}$. It might be that Marik's study featured unusually sick patients in the control group. Study participants are often unrepresentative of the general population and the intervention group may have been less likely to suffer an adverse outcome $^{19}$. This progress in sepsis care management is complicated by the fact that claims data has shown concurrently increasing sepsis incidence and decreasing mortality, so the literature is conflicted $^{20,21}$. It is possible that the increased incidence from claims data is due to increased reimbursement received by US hospitals for sepsis compared to other diseases, and patients are being misdiagnosed. An increasing rate of misdiagnosis of patients that do not have sepsis increases the denominator of septic cases while mortality stays the same in the numerator, creating the illusion of declining mortality ${ }^{13,21}$.

Regarding the mortality of Marik's treatment group, we note the significant improvement in outcomes recommending the use of steroids in adults with septic shock by Annane et al. $(2018)^{22}$. Patients randomised to the steroid group $(n=614)$ showed a $6 \%$ absolute reduction in 90-day all-cause mortality when compared to placebo $(n=627)$. Therefore, the figures in Marik's patient cohort may have been unusual, but they are plausible.

There is no current body of evidence that suggests this bundle is dangerous. Indeed, the combination of vitamin $\mathrm{C}$ and thiamine with steroids would have to cause an attributable death once in every 10 sepsis patients - by a hitherto unimagined and novel mechanism - to negate the modelled benefits of its early adoption.

\section{Limitations}

Our study excluded the costs of bundle implementation, including training, labour and the potential for high costs of de-implementing an unsuccessful treatment. These would have increased the cost of bundle implementation, so the incremental cost difference of the Marik bundle may be understated. We noted that hydrocortisone, as part of the bundle, was found by Venkatesh et al. (2018) to speed up resolution of shock and reduce the need for blood transfusions, so the in-hospital cost-savings for the treatment group may also be understated ${ }^{16}$. There is also considerable uncertainty around the parameter estimates that are available. We did not have access to primary data, including clinical data on costs and transition probabilities for each patient, and were reliant on the literature.

\section{Bundle adoption decision}

An average hospital in the US may treat around 230 sepsis patients per year ${ }^{12}$. By implementing the bundle, it will spend an additional $\$ 528$ per patient, or $\$ 121,440$ per year. Conservative estimates from the scenario analysis shows cost savings of $\$ 32,644$ and a gain of 0.55 QALYs per patient. If the treatment was effective for just four patients out of 230 , or $1.7 \%$, it will have paid for itself in terms of total economic costs. Comparing implementing the sepsis bundle to other hospital-based treatment studies shows that for 230 patients, the bundle costs less than a tenth of a standard phase I clinical trial, which run from $\$ 1.4 \mathrm{~m}-\$ 6.5 \mathrm{~m}^{23}$.

The case for not adopting the Marik bundle has several components. Scientific and empirical evidence is thin, and a single-site Vitamin C trial showing remarkable results only to be proven ineffective after a multi-site RCT is a clinical trope. If the bundle was ineffective, health systems will have added an unnecessary load to clinicians and implementers, which would then have to be de-implemented. Administrators and clinicians 
may be less likely to adopt novel treatments in the future. Introducing the bundle on the current evidence may also set a bad precedent for novel treatments, eroding the authority of the presiding physician and giving more credence to largely unproven interventions. The proposal in this paper does not replace the need for the clarity provided by good science and empirical research, but these are not always immediately available. We provide an approach to explicitly guide the interim decisions that must be made under these circumstances.

The Marik bundle is a somewhat unusual case relative to most 'miracle' interventions later found to be ineffective. As the analysis shows, it is an extremely cheap treatment with the potential to reduce rates of mortality and kidney damage at no risk to the patient. The delay between publication of the pilot study and results of the large RCT due in mid-2018 could create substantial opportunity costs in dollars and lives, and while there is not perfect evidence, under the circumstances it might be sufficient for hospitals and health systems to choose whether to conduct their own trials and not only independently verify their results, but also publish their findings and improve the availability of evidence around the treatment bundle.

The implementation decision of the Marik bundle relies upon the willingness of health administrators to use the available evidence to influence policy. We attempted to make the bundle choice as intuitive as possible, using straightforward trade-offs, simple modelling techniques, and a realistic decision process.
Merlo et al. (2014) showed that for research to be accessible to decision-makers, it must be contextually relevant, contain little jargon, and put the terms of the implementation decision into terms that specify a trade-off that will be familiar to decision-makers ${ }^{24}$. If the decision maker is unwilling to use the best available evidence now, but rather wishes to wait for definitive evidence they are risking incurring large costs for health care systems and for the patients it serves.

\section{Conclusion}

An explicit analysis of uncertain clinical outcomes is a useful adjunct to guide decision making where there is clinical ambiguity. This approach offers a valid alternative to the default of clinical inactivity when faced with uncertainty.

\section{Data availability}

Dataset 1. Sepsis Distributions Table: Data on distributions used in the analysis. 10.5256/f1000research.14619.d20141125

\section{Competing interests}

The authors have no financial interests, affiliations, gifts, grants, equipment or drugs to disclose.

\section{Grant information}

The author(s) declared that no grants were involved in supporting this work.
1. Liu V, Escobar GJ, Greene JD, et al.: Hospital deaths in patients with sepsis from 2 independent cohorts. JAMA. 2014; 312(1): 90-2. PubMed Abstract | Publisher Full Text

2. Alobaidi R, Basu RK, Goldstein SL, et al.: Sepsis-associated acute kidney injury. Semin Nephrol. 2015; 35(1): 2-11.

PubMed Abstract | Publisher Full Text | Free Full Text

3. Marik PE, Khangoora V, Rivera R, et al:: Hydrocortisone, Vitamin $\mathrm{C}$ and Thiamine for the Treatment of Severe Sepsis and Septic Shock: A Retrospective BeforeAfter Study. Chest. 2017; 151(6): 1229-38. PubMed Abstract | Publisher Full Text

4. Rezane S: The Marik Protocol: Have We Found a "Cure" for Severe Sepsis and Septic Shock? R.E.B.E.L. EM; 2017. Reference Source

5. Farkas J: PulmCrit - Metabolic sepsis resuscitation: the evidence behind Vitamin C. emcrit.org; 2017. Reference Source

6. SGEM: SGEM\#174: Don't Believe the Hype - Vitamin C Cocktail for Sepsis. thesgem.com; 2017. Reference Source

7. Arefian $\mathrm{H}$, Heublein $\mathrm{S}$, Scherag A, et al.: Hospital-related cost of sepsis: A systematic review. J Infect. 2017; 74(2): 107-17. PubMed Abstract | Publisher Full Text

8. USRDS: 2017 ADR Reference Table K: Economic Costs of ESRD. 2017. Reference Source

9. Torrance GW: Measurement of health state utilities for economic appraisal. $J$ Health Econ. 1986; 5(1): 1-30. PubMed Abstract | Publisher Full Text

10. Cuthbertson $\mathrm{BH}$, Roughton S, Jenkinson D, et al:: Quality of life in the five years after intensive care: a cohort study. Crit Care. 2010; 14(1): R6. PubMed Abstract | Publisher Full Text | Free Full Text

11. Johansen KL, Smith MW, Unruh ML, et al.: Predictors of health utility among 60-day survivors of acute kidney injury in the Veterans Affairs/National Institutes of Health Acute Renal Failure Trial Network Study. Clin J Am Soc Nephrol. 2010; 5(8): 1366-72.

PubMed Abstract | Publisher Full Text | Free Full Text

12. Angus DC, Linde-Zwirble WT, Lidicker J, et al.: Epidemiology of severe sepsis in the United States: analysis of incidence, outcome, and associated costs of care. Crit Care Med. 2001; 29(7): 1303-10. PubMed Abstract | Publisher Full Text

13. Rhee C, Dantes R, Epstein L, et al:: Incidence and Trends of Sepsis in US Hospitals Using Clinical vs Claims Data, 2009-2014. JAMA. 2017; 318(13): $1241-9$.

PubMed Abstract | Publisher Full Text | Free Full Text

14. Mallat J, Lemyze M, Thevenin D: Do not forget to give thiamine to your septic shock patient! J Thorac Dis. 2016; 8(6): 1062-6.

PubMed Abstract | Publisher Full Text | Free Full Text

15. Ohno S, Ohno Y, Suzuki N, et al:: High-dose vitamin C (ascorbic acid) therapy in the treatment of patients with advanced cancer. Anticancer Res. 2009; 29(3): 809-15.

PubMed Abstract

16. Venkatesh B, Finfer S, Cohen J, et al:: Adjunctive Glucocorticoid Therapy in Patients with Septic Shock. N Engl J Med. 2018; 378(9): 797-808. PubMed Abstract | Publisher Full Text

17. Stevenson EK, Rubenstein AR, Radin GT, et al:: Two decades of mortality trends among patients with severe sepsis: a comparative meta-analysis*. Crit Care Med. 2014; 42(3): 625-31.

PubMed Abstract | Publisher Full Text | Free Full Text 
18. Levy MM, Rhodes A, Phillips GS, et al:: Surviving Sepsis Campaign: association between performance metrics and outcomes in a 7.5-year study. Intensive Care Med. 2014; 40(11): 1623-33.

PubMed Abstract | Publisher Full Text

19. Kalata $P$, Martus $P$, Zettl $H$, et al.: Differences between clinical trial participants and patients in a population-based registry: the German Rectal Cancer Study vs. the Rostock Cancer Registry. Dis Colon Rectum. 2009; 52(3): 425-37. PubMed Abstract | Publisher Full Text

20. Glück T: Mortality from Severe Sepsis Decreasing in the U.S. www.jwatch.org: NEJM. 2013 Reference Source

21. Dressler DD: Is Sepsis Incidence and Mortality Changing in U.S. Hospitals? www.jwatch.org: NEJM. 2017.

Reference Source
Annane D, Renault A, Brun-Buisson C, et al:: Hydrocortisone plus Fludrocortisone for Adults with Septic Shock. N Engl J Med. 2018; 378(9): 809-18. PubMed Abstract | Publisher Full Text

23. Sertkaya A, Wong HH, Jessup A, et al:: Key cost drivers of pharmaceutical clinical trials in the United States. Clin Trials. 2016; 13(2): 117-26. PubMed Abstract | Publisher Full Text

24. Merlo G, Page K, Ratcliffe J, et al.: Bridging the gap: exploring the barriers to using economic evidence in healthcare decision making and strategies for improving uptake. Appl Health Econ Health Policy. 2015; 13(3): 303-9. improving uptake. Appl Health Econ Healt

25. Blythe R, Cook D, Graves N: Dataset 1 in: Scepticaemia: The impact on the health system and patients of delaying new treatments with uncertain evidence; a case study of the sepsis bundle. F1000Research. 2018 Data Source 


\section{Open Peer Review}

\section{Current Peer Review Status:}

\section{Version 1}

Reviewer Report 04 June 2018

https://doi.org/10.5256/f1000research.15909.r34658

(C) 2018 Visser A. This is an open access peer review report distributed under the terms of the Creative Commons Attribution License, which permits unrestricted use, distribution, and reproduction in any medium, provided the original work is properly cited.

\section{Adam Visser}

Toowoomba Hospital, Toowoomba, QId, Australia

This fascinating paper uses a simple health economics model to argue that delaying the widespread implementation of the Vitamin C protocol as described by Marik and colleagues would lead to the loss of enormous potential savings to the health industry. The argument is well presented, easily followed, and sound, with some exceptions.

There is likely to be some inaccuracy in the figures presented. The authors state that there are 1.5 million cases of sepsis in the United States yearly (no reference supplied, but data easily available via the $C D C$ ). Whilst there may indeed be this number who meet the recently updated definition of sepsis, this large cohort of patients are different to those who have a mortality rate of $40 \%$ with standard care. This group, as described in Marik's paper, are a much sicker population. Their number is likely to be significantly fewer than 1.5 million. Whilst it is not known whether Vitamin C may have benefits in a less sick population, it is unlikely that 'early adopters' of this therapy will consider it in those that are not towards the severe end of the sepsis spectrum. Reducing the exposed population in the model from 1.5 million will reduce the proposed savings by a proportional amount.

Similarly, the quoted incidence of requirement for renal replacement therapy is overstated. It is true that the Marik Vitamin C-treated group had an incidence of RRT-requiring AKI of 9.7\%. However, the Marik paper does not state that these patients all went on to require ongoing RRT for ESRF (at the authors' quoted cost of $\$ 77,000$ per year). The majority of patients who require RRT in the setting of sepsis-induced AKI will recover their renal function either completely, or enough that they do not require ongoing dialysis. Again, reducing the stated financial benefits of this aspect of the Vitamin C treatment will reduce the long-term economic attractiveness of the therapy.

Overall however, the authors are correct in their statement that the economic implications of adopting the 'bundle' will be positive. Furthermore, the economic implications should be secondary to the beneficial effects the treatment may have on mortality. Again, this therapy is unlikely to be offered to 1.5 million patients per year, but whatever the number, those who receive 
it are likely to benefit.

A major strength of the paper is their use of a more pessimistic scenario (scenario 1) than published in the Marik study to demonstrate the robustness of their figures. The Marik-stated improvement in mortality, from $40 \%$ to $8 \%$, is likely to not be repeated in larger studies, as it is simply too great to be plausible. Reducing the relative risk reduction such that mortality is reduced from $21 \%$ to $8 \%$ may be more realistic, and yet the economic figures still add up to considerable savings.

It would perhaps also be useful to see a model where baseline mortality of $40 \%$ (ie the very sick cohort from Marik) have their mortality reduced to, say, 30\% (ie a more plausible magnitude of effect from Vitamin C).

The authors have done well to pick this particular therapy to demonstrate their model. The Vitamin C bundle is unique in recent years, in that it is a cheap and seemingly safe therapy. Their model is unlikely to be able to be used for supporting other therapies that emerge in coming years, given the likelihood that these therapies will come from the pharmaceutical industry and have large price tags attached.

Is the work clearly and accurately presented and does it cite the current literature? Yes

Is the study design appropriate and is the work technically sound? Yes

Are sufficient details of methods and analysis provided to allow replication by others? Yes

If applicable, is the statistical analysis and its interpretation appropriate? Partly

Are all the source data underlying the results available to ensure full reproducibility? Yes

Are the conclusions drawn adequately supported by the results? Yes

Competing Interests: No competing interests were disclosed.

Reviewer Expertise: Critical care medicine, sepsis

I confirm that I have read this submission and believe that I have an appropriate level of expertise to confirm that it is of an acceptable scientific standard.

Reviewer Report 11 May 2018

https://doi.org/10.5256/f1000research.15909.r33910 
(C) 2018 Marik P. This is an open access peer review report distributed under the terms of the Creative Commons Attribution License, which permits unrestricted use, distribution, and reproduction in any medium, provided the original work is properly cited.

\section{Paul E. Marik}

Division of Pulmonary and Critical Care Medicine, Eastern Virginia Medical School, Norfolk, VA, USA

This is an outstanding paper that objectively evaluates the potential benefit of the vitamin C protocol for sepsis. This evaluation was impeccably conducted and reported. The impact of this paper is significant.

A few comments that will improve the paper:

1.The authors state "The delay between publication of the pilot study and results of the large RCT due in mid-2018 could create substantial opportunity costs". This study which the authors cite is the NIH funded study due for completion in mid-2018 which is investigating vitamin c (ALONE) and not the combination AND in patients with ARDS (not specifically sepsis). Secondly, this study is not powered for a mortality difference. Currently according to Clintrials.gov there are 8 RCTS testing the combination and 3 testing Vitamin $C$ alone. This suggests that it may take at least until the end of 2019 before the results of any of these studies are available and we can make firm conclusions.

2. The authors refer to the "sepsis bundle"; I would avoid using this term as it will create confusion with the Surviving Sepsis Bundle.. widely known as the Sepsis Bundle. I would call it the Vitamin C protocol, the Marik Protocol, or something similar.

3. Approximately $50 \%$ of sepsis survivors develop the post-sepsis syndrome, defined as severe physical and cognitive dysfunction not unlike PTSD. This syndrome is associated with enormous suffering and high costs. It is likely that the Marik Protocol will reduce the incidence and severity of this syndrome. While the cost savings are difficult to quantitate, I think that this proposition should be mentioned in the discussion section.

Is the work clearly and accurately presented and does it cite the current literature? Yes

Is the study design appropriate and is the work technically sound?

Yes

Are sufficient details of methods and analysis provided to allow replication by others? Yes

If applicable, is the statistical analysis and its interpretation appropriate? Yes

Are all the source data underlying the results available to ensure full reproducibility? Yes

Are the conclusions drawn adequately supported by the results? 
Yes

Competing Interests: I am the first author of the primary study under review.

I confirm that I have read this submission and believe that I have an appropriate level of expertise to confirm that it is of an acceptable scientific standard.

The benefits of publishing with F1000Research:

- Your article is published within days, with no editorial bias

- You can publish traditional articles, null/negative results, case reports, data notes and more

- The peer review process is transparent and collaborative

- Your article is indexed in PubMed after passing peer review

- Dedicated customer support at every stage

For pre-submission enquiries, contact research@f1000.com 